\title{
The lower limit of the first frequency of natural vibrations externally statically indeterminate truss: analytical solution
}

\author{
Mikhail Kirsanov", and Alexander Shirokov \\ ${ }^{1}$ National Research University "Moscow Power Engineering Institute," Moscow, Russia
}

\begin{abstract}
A scheme of a statically determinate planar truss with two additional supports duplicating the main ones is proposed. The formula for the dependence of the lower estimate of the first natural frequency on the number of panels is obtained. The solution is compared with the numerical one. Determination of the forces in the rods by the method of cutting out the nodes and with all the transformations performed in the Maple computer mathematics system. The high accuracy of the result is shown with a large number of panels.
\end{abstract}

\section{Introduction}

Calculation of the spectra of natural vibrations of structures, along with the calculation of strength and stability, is of great importance for ensuring the operability of structures. The first (fundamental) frequency in the natural frequency spectrum is the most important. Finding the entire spectrum requires the use of numerical methods [1,2]. There are two approximate estimates for determining the first frequency. Below is Dunkerley's estimate [3-5], above - Rayleigh [6]. The calculation formulas in these methods are quite simple, so analytical approaches are applicable here. In this paper, we consider calculating the first frequency of a regular truss by the Dunkerley method. The task is to obtain an analytical expression for the dependence of the first frequency on the size and weight of the truss and the number of panels. The peculiarity of the proposed scheme of the truss is that additional supports are introduced into the scheme, making this truss outwardly statically indeterminate. It is impossible to determine the support reactions regardless of the forces in the rods in such a truss. Static analysis and formulas for the deflection of such trusses depending on the number of panels are given in the handbook [7]. The problems of the existence of regular statically determinate rod systems and their calculation were raised in the works $[8,9]$.

Analysis of the spectra of natural vibrations of building structures is in demand in solving problems of seismic safety [10-14] and in optimization problems [14-17].

\footnotetext{
${ }^{*}$ Corresponding author: c216@ya.ru
} 


\section{Methods}

\subsection{The truss scheme}

Consider a girder-type truss with a diagonal lattice. The inertial properties of the truss are modeled by identical weights located at the nodes of the lower belt. A truss with $n$ panels in half a span contains $\mu=8 n+12$ rods. Each panel contains two rods of length in chords and two in a cruciform lattice. The side panels are shortened and have additional movable support. The truss is symmetrical. Ignoring horizontal displacements, consider only vertical vibrations of the weights. In this case, the number of degrees of freedom of the cargo system of the truss is equal to $N=2 n-3$. The height of the truss is $3 h$. Despite the external static uncertainty, analytical methods are available for this truss to find the dependences of deformations and forces on the number of panels.

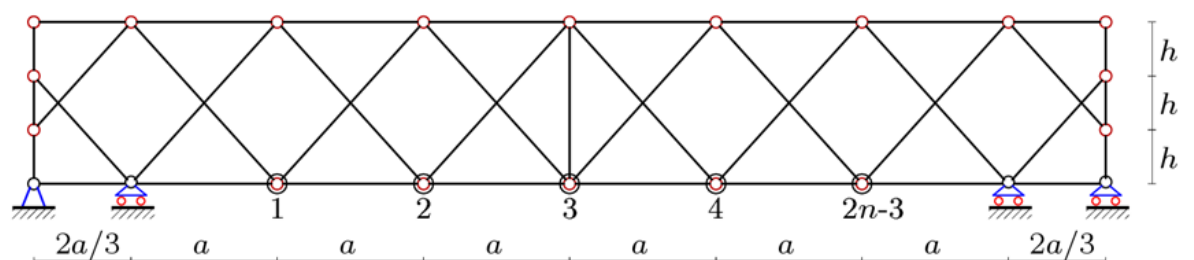

Fig. 1. Truss, $n=4$.

\subsection{Dunkerley method}

Consider an approximate solution using the Dunkerley method. The lower estimate of the first vibration frequency is given by the formula:

$$
\omega_{D}^{-2}=\sum_{k=1}^{N} \omega_{k}^{-2}
$$

where $\omega_{k}$ is the vibration frequency of one mass located at the node of the lower belt. The dynamic equation of vibrations of one mass has the form:

$m \ddot{y}_{k}+d_{k} y_{k}=0$,

where $d_{k}$ is the stiffness coefficient ( $k$ is the mass number), $y_{k}$ is the vertical displacement of the mass, and $\ddot{y}_{k}$ is the acceleration. Hence the expression for the vibration frequency of a single mass $\omega_{k}=\sqrt{d_{k} / m}$ (partial frequency) follows. The stiffness coefficient is determined by the Maxwell-Mohr's formula:

$$
\delta_{k}=1 / d_{k}=\sum_{\alpha=1}^{\mu-5}\left(\tilde{S}_{\alpha}^{(k)}\right)^{2} l_{\alpha} /(E F)
$$

Here it is indicated $\tilde{S}_{\alpha}^{(k)}$ the forces in the member with a number $\alpha$ from the action of a single vertical force applied to the node, where the mass with the number $\mathrm{k}$ is located, where $E F$ is the stiffness of the bars, $l_{\alpha}$ is the length of the bar $\alpha$. The forces of five nondeformable support rods are not included in the total. According to (1) 


$$
\omega_{D}^{-2}=m \sum_{k=1}^{N} \delta_{k}=m \Delta_{n}
$$

To find forces in the elements, you can use the program in the Maple [18] system, which gives forces in an analytical form. The program uses the method of cutting nodes and solving the joint system of equilibrium equations for all nodes of the truss. The unknowns also include the reactions of the supports. The system matrix includes the values of the direction cosines of the forces found from the coordinates of the regular grid of nodes. The calculation of the sequence of trusses with an increasing number of panels shows that the type of the sum $\Delta_{n}$ does not change (property of the regularity of the system)

$$
\Delta_{n}=\left(C_{1, n} a^{3}+C_{2, n} c^{3}+C_{3, n} h^{3}\right) /\left(h^{2} E F\right)
$$

where $c=\sqrt{a^{2}+9 h^{2}}$ is the length of the brace. We have a sequence of formulas:

$$
\begin{aligned}
& \Delta_{2}=\left(a^{3}+c^{3}+54 h^{3}\right) /\left(18 h^{2} E F\right), \\
& \Delta_{3}=\left(19 a^{3}+11 c^{3}+108 h^{3}\right) /\left(18 h^{2} E F\right), \\
& \Delta_{4}=\left(673 a^{3}+225 c^{3}+1458 h^{3}\right) /\left(162 h^{2} E F\right), \\
& \Delta_{5}=\left(215 a^{3}+47 c^{3}+216 h^{3}\right) /\left(18 h^{2} E F\right), \\
& \Delta_{6}=\left(2477 a^{3}+365 c^{3}+1350 h^{3}\right) /\left(90 h^{2} E F\right) .
\end{aligned}
$$

The general terms of the sequences of coefficients at $a^{3}, c^{3}$ and $h^{3}$ are obtained from solving the recurrent equations using the rgf_findrecur operator from the special genfunc package of the Maple system

$$
\begin{aligned}
& C_{1}=\left(64 n^{4}-256 n^{3}+574 n^{2}-636 n-45(-1)^{n}+135\right) / 1620, \\
& C_{2}=\left(6 n^{2}-12 n-(-1)^{n}+3\right) / 36 \\
& C_{3}=3(n-1) .
\end{aligned}
$$

Taking into account (2) and (3), we obtain the final formula for the lower boundary of the first natural vibration frequency of the structure:

$$
\omega_{D}=h \sqrt{\frac{E F}{m\left(C_{1} a^{3}+C_{2} c^{3}+C_{3} h^{3}\right)}}
$$

\subsection{Calculation of the spectrum of natural frequencies}

Consider an algorithm for calculating the entire frequency spectrum of a truss. The first (smallest) value of this spectrum is needed for comparison with the obtained analytical one. Differential equations of the dynamics of the system of loads have the form: 


$$
M_{N} \ddot{Y}+D_{N} Y=0
$$

where $Y=\left[y_{1}, y_{2}, \ldots, y_{N}\right]^{T}$ are the vertical displacements of the masses, $D_{N}$ is the stiffness matrix, $M_{N}$ is the diagonal matrix of inertia in size $N \times N$, and $\ddot{Y}$ is the acceleration vector. If the masses are the same, then the inertia matrix is expressed through the unit one $M_{N}=m I_{N}$. The compliance matrix $B_{N}$ is inverse to the stiffness matrix $D_{N}$. Its elements are determined in the same way as in the one-dimensional case by the Maxwell - Mohr formula:

$$
b_{i, j}=\sum_{\alpha=1}^{\mu-5} S_{\alpha}^{(i)} S_{\alpha}^{(j)} l_{\alpha} /(E F)
$$

Multiply (6) by $B_{N}$ the left. Taking into account the identity $\ddot{Y}=-\omega^{2} Y$, which is valid for small harmonic oscillations, the problem is reduced to the problem of matrix $B_{N}$ eigenvalues: $B_{N} Y=\lambda Y$, where $\lambda=1 /\left(m \omega^{2}\right)$ is the eigenvalue of the matrix $B_{N}, \omega$ is the natural frequency of oscillations. This problem can be solved only numerically.

\section{Results and Discussion}

We estimate the error of the formula (5) from comparison with the numerical solution of the problem on the eigenvalues of the matrix. The eigenvalues of the matrix are determined using the Eigenvalues operator from the LinearAlgebra package of the Maple system. Graph (2) compares the curves of the dependence of the first frequency, obtained numerically and according to formula (5). The elastic modulus for steel rods is adopted: $E=2 \cdot 10^{5} \mathrm{MPa}$, the cross-sectional areas of the rods are equal: $F=40.5 \mathrm{sm}^{2}$. In the nodes of the lower belt, masses $\mathrm{m}=1000 \mathrm{~kg}$ are located. For plotting the graphs, the following dimensions were taken: $a=5 \mathrm{~m}, h=3 \mathrm{~m}$. The curve constructed by the analytical solution (5) turns out to be slightly lower than the numerical solution. The accuracy of the obtained analytical solution increases with the number of panels. This can be seen more clearly if we introduce the relative error $\varepsilon=\left(\omega_{1}-\omega_{D}\right) / \omega_{1}$. If we do not take into account the first section corresponding to unrealistically small numbers of panels, then we can conclude that an increase in the number of panels leads to an increase in the accuracy of the obtained formula (5). 


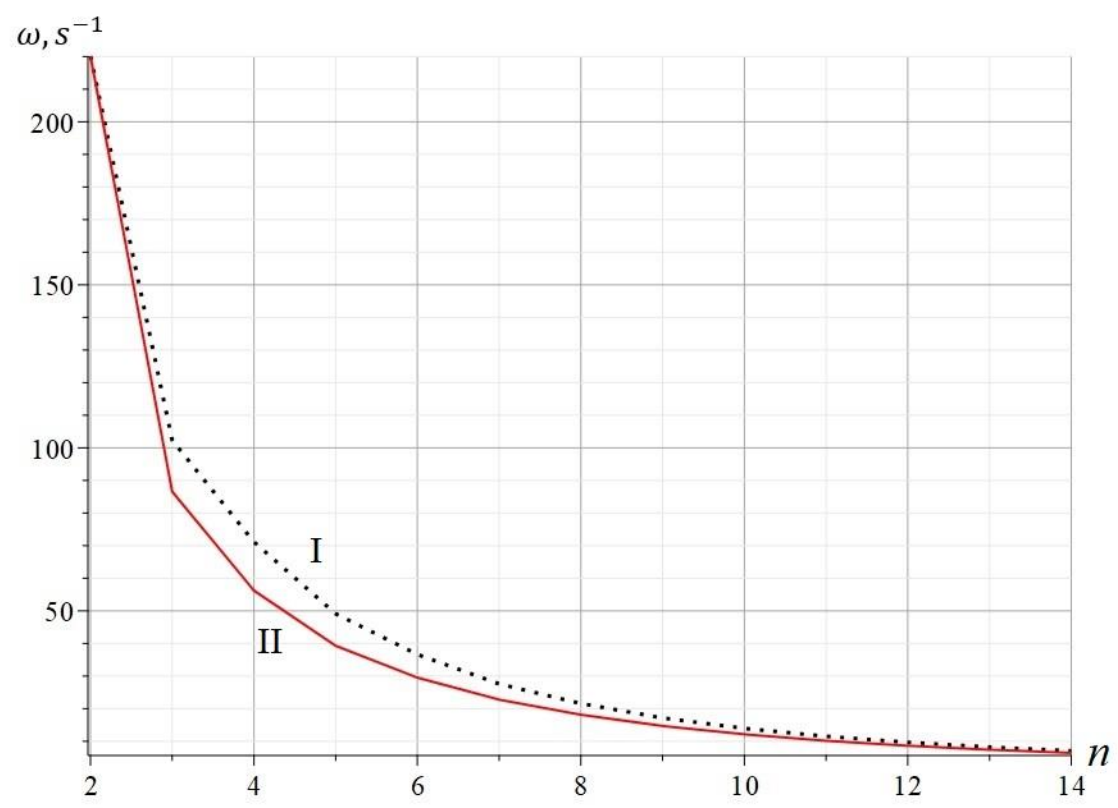

Fig. 2. Frequency dependence on the number of panels; I is numerical solution $\omega_{1}$; II is analytical assessment $\omega_{D}$.

Calculations show that the choice of dimensions, stiffness, and mass has almost no effect on the accuracy of the lower estimate. Only the number of panels has the greatest impact.

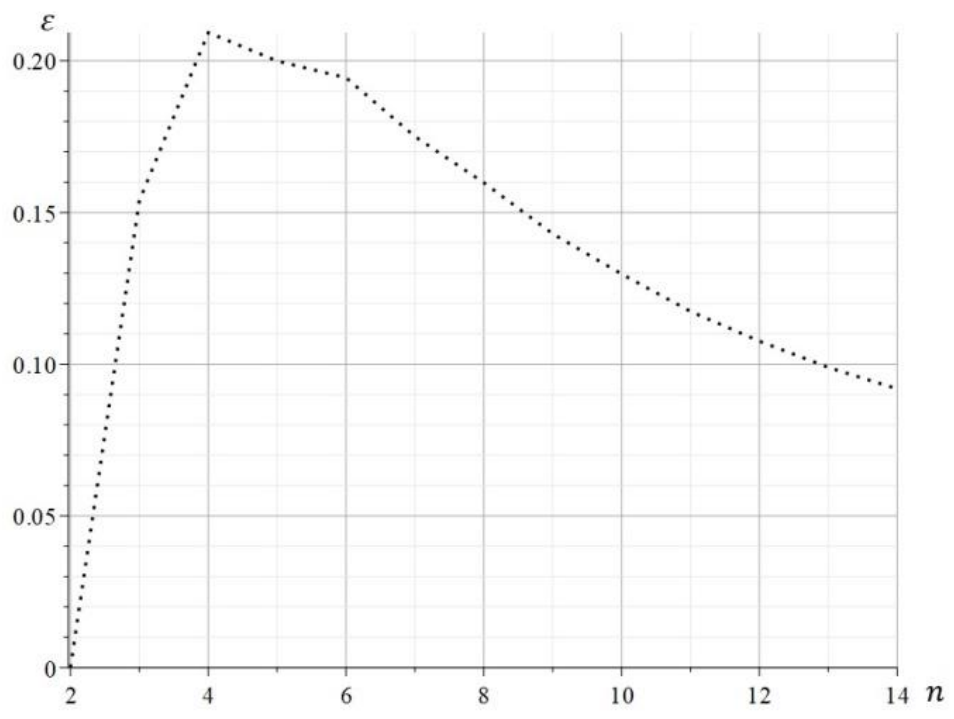

Fig. 3. The error of Dunkerley estimation depending on the number of panels

Depending on the number of panels, the solution error changes monotonically from $21 \%$ at $n=4$ to $9 \%$ at $n>14$. 
On the basis of the described technique for obtaining the lower frequency limit, the authors also attempted to find the upper limit of the first frequency in an analytical form using the Rayleigh energy method [6]. It is known that the Rayleigh method is more accurate than the Dunkerley method. However, the final formula turned out to be too cumbersome for practical use.

The found solution to the problem for estimating the first truss frequency for an arbitrary number of panels turned out to be quite simple. It does not contain sums and series and does not involve the use of special functions. Polynomials in the number of panels through which the coefficients are calculated have a degree of at most four. The formulas can be used to estimate the frequency of oscillations of a truss with a very large number of rods, that is, in those cases where the accumulation of computational errors is most likely, and difficulties arise with the amount of computations. The obtained estimate can also be used for a truss with different stiffness of bar elements. It is enough to introduce two correction factors $\gamma_{h}$ and $\gamma_{c}$ for the stiffness of the lattice rods. The values of the coefficients (4) will not change, and formula (3) in this case takes the form

$$
\Delta_{n}=\left(C_{1, n} a^{3}+C_{2, n} c^{3} / \gamma_{c}+C_{3, n} h^{3} / \gamma_{h}\right) /\left(h^{2} E F\right)
$$

\section{Conclusion}

The main results of the work are as follows:

1. A scheme of a truss with additional supports is proposed. Despite the external static indeterminacy, the design allows an analytical solution to the problem.

2. An explicit dependence of the main frequency of oscillations of the truss on the number of panels was obtained.

3. Comparison with the numerical solution of the eigenvalue problem has shown the high accuracy of the analytical solution found for a large number of panels.

\section{Acknowledgements}

The investigation was carried out within the framework of the project "Dynamics of light rod structures of manipulators" with the support of a grant from NRU "MPEI" for implementation of scientific research programs "Energy", "Electronics, Radio Engineering and IT", and "Industry 4.0, Technologies for Industry and Robotics in 2020-2022".

\section{References}

1. Khodzhaev D.A., Abdikarimov R.A., Vatin N.I. Nonlinear oscillations of a viscoelastic cylindrical panel with concentrated masses. (2015), URL: https://elibrary.ru/item.asp?id=38224827 (date of application: 5.03.2021).

2. Vatin N., Ivanov Ay., Rutman Y., Chernogorskiy S., Shvetsov K. Earthquake engineering optimization of structures by economic criterion, Magazine of Civil Engineering. 8(76). pp. 67-83. (2017), DOI:10.18720/MCE.76.7.

3. Low K.H. Modified Dunkerley formula for eigenfrequencies of beams carrying concentrated masses, International Journal of Mechanical Sciences, 42(7). pp. 12871305, (2000), DOI:10.1016/S0020-7403(99)00049-1.

4. Levy C. An iterative technique based on the Dunkerley method for determining the natural frequencies of vibrating systems. Journal of Sound and Vibration, 150(1). pp. 111-118, (1991), DOI:10.1016/0022-460X(91)90405-9. 
5. Trainor G.S., Shah A.H., Popplewell N. Estimating the fundamental natural frequency of towers by Dunkerley's method. Journal of Sound and Vibration, 109(2), pp. 285292, (1986), DOI:10.1016/S0022-460X(86)80009-8.

6. Low K.H. Frequencies of beams carrying multiple masses: Rayleigh estimation versus eigenanalysis solutions. Journal of Sound and Vibration, 268(4), pp. 843-853, (2003), DOI:10.1016/S0022-460X(03)00282-7.

7. Kirsanov M. Planar Trusses: Schemes and Formulas - Cambridge Scholars Publishing. URL: https://www.cambridgescholars.com/product/978-1-5275-3531-2 (date of application: 27.02.2021).

8. Hutchinson R.G., Fleck N.A. The structural performance of the periodic truss. Journal of the Mechanics and Physics of Solids. 54(4), pp. 756-782, (2006), DOI:10.1016/j.jmps.2005.10.008.

9. Hutchinson R.G., Fleck, N.A. Microarchitectured cellular solids - the hunt for statically determinate periodic trusses, ZAMM Zeitschrift fur Angewandte Mathematik und Mechanik, 85(9), pp. 607-617. (2005), DOI:10.1002/zamm.200410208.

10. Kilikevicius A., Fursenko A., Jurevicius M., Kilikeviciene K., Bureika, G. Analysis of parameters of railway bridge vibration caused by moving rail vehicles, Measurement and Control 52(9-10), pp. 1210-1219, United Kingdom, (2019), DOI: $10.1177 / 0020294019836123$.

11. Martins A.M.B., Simões L.M.C., Negrão H.J.O. Optimization of extradosed concrete bridges subjected to seismic action. Computers and Structures. 245, (2021), DOI:10.1016/j.compstruc.2020.106460.

12. Pekcan G., Itani A.M., Linke C. Enhancing seismic resilience using truss girder frame systems with supplemental devices. Journal of Constructional Steel Research. 94, pp. 23-32, (2014), DOI:10.1016/j.jcsr.2013.10.016.

13. Resatalab S., Ahmadi M.T., Alembagheri M. Seismic response sensitivity analysis of intake towers interacting with dam, reservoir and foundation, Magazine of Civil Engineering. 98(7), pp. 9901-9901, (2020) DOI:10.18720/MCE.99.1. URL: https://engstroy.spbstu.ru/article/2020.99.1 (date of application: 27.02.2021).

14. Tarasov, V.A. Double Seismic Insulation System of Turbine Unit Foundation. Construction of Unique Buildings and Structures. 91(6). pp. 9101-9101. (2020), DOI:10.18720/CUBS.91.1.

15. Qiu, Z., Liu, D., Wang, L., Xia, H. Scale-span stress-constrained topology optimization for continuum structures integrating truss-like microstructures and solid material, Computer Methods in Applied Mechanics and Engineering, 355, pp. 900925, (2019), DOI:10.1016/j.cma.2019.07.002.

16. A Kaveh. Cyclical parthenogenesis algorithm for layout optimization of truss structures with frequency constraints. Eng. Optim. 49(8), pp. 1317-1334, (2017)

17. Vorobev O.V. Bilateral Analytical Estimation of the First Frequency of a Plane Truss, Construction of Unique Buildings and Structures, 92(7). pp. 9204-9204, (2020), DOI:10.18720/CUBS.92.4.

18. Rapp B.E. Introduction to Maple. Microfluidics: Modelling, Mechanics and Mathematics. pp. 9-20, Elsevier, (2017) 